\title{
SCOT is a vital enzyme for tumors: With reference to Carney Triad Cancers and the ketogenic diet
}

\author{
Maurice Israël ${ }^{*}$ and Laurent Schwartz ${ }^{2}$ \\ ${ }^{1}$ Institut Alfred Fessard CNRS, 2 Av. Terrasse, 91190 Gif sur Yvette, France \\ ${ }^{2}$ Assistance Publique des Hôpitaux de Paris, 3 Av. Victoria, 75004, Paris, France
}

\begin{abstract}
Mitotic tumor cells have to synthetize fatty acids and lipid membranes; fatty acid synthesis forms a malonylCoA intermediate, which inhibits the mitochondrial fatty acid carnityl transporter and closes automatically their degradation and beta-oxidation into acetylCoA. Since the glycolytic supply of acetylCoA is OFF as well, (a persistent phosphorylation inhibits pyruvate kinase and pyruvate dehydrogenase); tumor cells become vitally dependent of ketolysis and of the specific ketolytic enzyme: succinyl-CoA: 3-oxoacid-CoA transferase (SCOT) for making acetylCoA in their mitochondria. We discuss the supply of the two substrates driving this enzyme: the first, succinylCoA, in relation to succinodehydrogenase (SDH)-deficient cancers of the Carney Triad; and the second, acetoacetic acid, in relation to the ketogenic diet.

Acetohydroxamic acid a non-toxic inhibitor for the ketolytic enzyme was in use for other medical indications; it may then block the tumor. Further, we earlier showed a regression of tumors after slowing down the citrate mitochondrial efflux, and inhibiting ATP citrate lyase, with lipoic acid-hydroxycitrate mixtures. However, tumor cells develop a salvage pathway by incorporating external acetate into the lipogenic pathway, via a cytosolic acetylCoA synthetase; allicine and orotic acid inhibit this enzyme.
\end{abstract}

\section{Introduction}

Tumor cells have to synthetize fatty acids and lipid membranes, which automatically turns off the degradation of fatty acid and this source of mitochondrial acetylCoA. Indeed, malonylCoA, the product of acetylCoA carboxylase, which forms along the fatty acid synthesis pathway, blocks the carnityl transporter of fatty acids and their mitochondrial beta-oxidation. We also know that the glycolytic supply of acetylCoA is not operational. Indeed, the works of Eigenbrodt and his team identified the tumor M2 Pyruvate kinase, its persistent phosphorylation closes the last step of glycolysis [1], and the supply of pyruvate, to pyruvate dehydrogenase, which is itself inhibited by phosphorylation; a bottleneck at the end of glycolysis closes the glycolytic acetylCoA supply to the Krebs cycle. The only possible way to form acetylCoA in tumor cell mitochondria will come from the ketolysis of ketone bodies produced in the liver by ketogenesis. Hence, tumor cells become vitally dependent of ketolysis and of the specific ketolytic enzyme succinyl-CoA: 3-oxoacid-CoA transferase (SCOT) to form acetylCoA in their mitochondria [2]. SCOT has two substrate entries, succinylCoA and acetoacetate a ketone body. The enzyme transfers the CoA of succinylCoA on acetoacetate, forming acetoacetylCoA. The latter gives then acetylCoA via the mitochondrial thiolase. This will start the citrate condensation reaction of the Krebs cycle.

There are tumor cells that display mutations of one of the four subunits of succinic acid dehydrogenase (SDH) they become unable to convert succinate into fumarate, the accumulated succinate will then drive succinylCoA sythetase, the preceding enzyme of the Krebs cycle, in the reverse direction forming more succinylCoA. Evidently, this mutation is favorable to the tumor cell since it boosts the activity of SCOT forming more acetoacetylCoA, and acetylCoA. A tumor cell that would escape from the immune system will become able to use more efficiently the ketolytic route for making acetylCoA in its mitochondria, via SCOT and develop. However, SCOT requires a second substrate: acetoacetate, it comes with the ketogenic diet, which precisely forms this ketone body for making acetylCoA in tumor cells. With the glycolytic and fatty acid sources of acetylCoA closed, the ketolytic supply becomes vital for tumor cells and the SDH mutation strengthens this supply, in association with the ketogenic diet, there is a gain of function allowing their development. The tumors with this SDH mutation belong to the Carney tirad [3] it includes SDH-deficient gastrointestinal intersticial tumors; SDH- deficient paraganglioma and pulmonary chondroma; SDH-deficient renal carcinoma. There are also pheochromocytomas, and probably more. The non-hereditary SDH mutations make more secure the supply of mitochondrial acetylCoA in tumor cells via SCOT, particularly if the liver receives catabolic glucagon signals for making ketone bodies, which is beneficial to tumor cells or if the ketogenic diet rich in fat helps their formation. This should call attention on this diet often prescribed in cancer. In his review, Klement [4] reminds that Brünings was first to use it. Initially, he wanted to limit the supply of glucose to tumors, following the discovery of Warburg on glucose to lactate conversion in presence of oxygen. In his trial, Brünings provokes an insulin-induced hypoglycemia; at the beginning of the trial, tumors did regress. In fact, Insulin injections inhibit glucagon release and

${ }^{\star}$ Correspondence to: Maurice Israël, 2 Av. Aristide Briand, 91440 Bures sur Yvette, France, E-mail: mauisrael@wanadoo.fr

Key words: Succinyl-CoA: 3-oxoacid-CoA transferase (SCOT), Succinodehydrogenase (SDH), SDH-deficient tumors, Carney triad, ketogenic diet, acetylCoA synthetase

Received: February 07, 2020; Accepted: February 20, 2020; Published: February 24,2020 
lower ketone bodies and acetoacetate, which deprives SCOT of its substrate, and tumor cells of acetylCoA. However, since patients take the high fat ketogetic diet it overcomes with time, the effects of insulin injections and ketone bodies increase, while one observes after a few weeks a disappointing rebound of tumors. Presumably, with the supply of beta hydroxybutyrate then acetoacetate, SCOT could make again mitochondrial acetylCoA for tumor cells. In the same line we know that glutaminolysis and glutamic acid supply is an essential process in tumor cell metabolism, glutamate is shuttled in mitochondria in exchange of aspartate, in mitochondria glutamate transamination gives back alpha ketoglutarate, while oxaloacetate forms aspartate. Well, alpha ketoglutarate the substrate of alphaketoglutarate dehydrogenase forms succinylCoA the substrate of SCOT. There are pharmacological projects for inhibiting glutaminolysis, this should deprive SCOT of one of its substrates, it should then be logic to suppress the second substrate as well, and to avoid the ketogenic diet. It seems to us essential to find a SCOT inhibitor. The Brenda chemical database gives a list of inhibitors, most are toxic (dinitrophenyl acetate difluorosuccinate), others (acetylimidazole, $\mathrm{N}$-acetylcysteamine could be tested). The work of Picard and Jenks [5] on the active center of SCOT indicates a particularly interesting compound, acetohydroxamic acid, which inhibits the enzyme-substrate complex. Acetohydroxamic acid is available as tablets under the name of lithostat; it fights kidney stones. Since SCOT inhibition by acetohydroxamic acid is a central point, we will summarize the inhibitory mechanism studied by Pickart and Jencks. In the active center of SCOT, the gamma carboxyl of a glutamyl residue reacts with the substrate succinylCoA and forms a thioester bond with $\mathrm{CoA}$ in the enzyme-substrate complex. The thioester then transfers the $\mathrm{CoA}$ to acetoacetate. The inhibitor acetohydroxamic acid inactivates the thioester of the enzyme-substrate complex, the most probable structure involving a bond between the enzyme carboxyl group and the hydroxylamine oxygen atom of acetohydroxamic acid. The inhibition is partly reversible by increasing the CoA concentration. Acetohydroxamic acid, which is a prescription for other medical indications, is less toxic than many inhibitors of SCOT. Our literature search found that a class of histone deacetylase (HDAC) inhibitors is the hydroxamic acid derivatives, such as suberoylanilide hydroxamic acid (SAHA), trichostatine, or vorinostat, [6] possibly salicylhydroxamic acid also. Most of these HDAC inhibitors have anticancer properties. Presumably, they inhibit SCOT, but the experimental context focused on HDAC and did not mention an effect on SCOT. However, the expected decrease of mitochondrial acetylCoA via SCOT inhibition, might indeed explain the anticancer effects. The citrate condensation then forms citrate, which will quit the mitochondria and feed the lipogenic route, via ATP citrate lyase, this enzyme splits back citrate in the cytosol forming acetylCoA and oxaloacetate. We earlier found that inhibiting ATP citrate lyase with hydroxycitrate and slowing down the citrate efflux by reducing NADH with lipoic acid, was effective in cancer [7]. Unfortunately, tumors develop a salvage pathway for surviving, by directly incorporating acetate in the lipogenic pathway, via a cytosolic acetylCoA synthetase [8] an enzyme normally found in neuromuscular cholinergic synapses. Compounds such as allicine from garlic and orotic acid from cow milk inhibit this enzyme see references in [2].

\section{Conclusion}

We hope to call the attention of clinicians and pharmacologists, on SCOT inhibition, which could be the vulnerable point of tumor cells. We also point that depriving the enzyme of its substrate coming with the ketogenic diet seems necessary; SCOT inhibition would then make it difficult for tumor cells to form mitochondrial acetylCoA. In addition, the inhibition of the acetylCoA synthetase a cytosolic salvage enzyme should hold back the development of the tumor.

\section{Conflicts of interest}

The authors declare no conflicts of interest.

\section{References}

1. Mazurek S, Eigenbrodt E (2003) The tumor metabolome. Anticancer Res 23: 11491154. [Crossref]

2. Israël M, Schwartz L (2020) The metabolic rewiring observed in cancer renders tumor cells dependent of ketone bodies and vulnerable to SCOT inhibition. Endocrinology Diabetes and Metabolism Journal 4: 1-13.

3. Carney JA, Sheps SG, Go VLW, Gordon H (1977) The triad of gastric leiomyosarcoma, functioning extra-adrenal paraganglioma, and pulmonary chondroma. New Engl J Med 296: 1517-1518. [Crossref]

4. Klement RJ (2018) Wilhelm Brünings' forgotten contribution to the metabolic treatment of cancer utilizing hypoglycemia and a very low carbohydrate (ketogenic) diet. J Tradit Complement Med 9: 192-200. [Crossref]

5. Pickart CM, Jencks WP (1979) Formation of stable anhydrides from CoA transferase ans hydroxamic acids. The Journal of Biological Chemistry 254: 9120-9129.

6. Pal D, Saha S (2012) Hydroxamic acid - A novel molecule for anticancer therapy. $J A d v$ Pharm Technol Res 3: 92-99. [Crossref]

7. Schwartz L, Abolhassani M, Guais A, Sanders E, Steyaert JM, et al. (2010) A combination of alpha lipoic acid and calcium hydroxycitrate is efficient against mouse cancer models: preliminary results. Oncol Rep 23: 1407-1416. [Crossref]

8. Comerford SA, Huang Z, Du X, Wang Y, Cai L, et al. (2014) Acetate dependence of tumors. Cell 159: 1591-1602. [Crossref]

Copyright: (C2020 Israël M. This is an open-access article distributed under the terms of the Creative Commons Attribution License, which permits unrestricted use, distribution, and reproduction in any medium, provided the original author and source are credited. 